\title{
Studying Virtual Worlds as Medium for Telepresence Robots
}

\author{
Alex Juarez \\ Eindhoven University of \\ Technology \\ Den Dolech 2 \\ Eindhoven, Netherlands \\ acordova@tue.nl
}

\author{
Christoph Bartneck \\ University of Canterbury \\ HIT Lab NZ \\ Christchurch, New Zealand \\ christoph.bartneck@canterbury.ac.nz
}

\author{
L. M. G. Feijs \\ Eindhoven University of \\ Technology \\ Den Dolech 2 \\ Eindhoven, Netherlands \\ I.m.g.feijs@tue.nl
}

\begin{abstract}
This paper presents a study on the effects of using virtual worlds as medium for interaction with telepresence robots. The Prototype for Assisted Communication PAC4 is also introduced. This system connects virtual worlds with real robots, allowing virtual world users to access the robot capabilities in a telepresence scenario. A user experiment was conducted to evaluate the effects of PAC4 on the feeling of social presence experienced by virtual world users.
\end{abstract}

\section{Categories and Subject Descriptors}

J.2 [Computer Applications]: Physical Sciences and Engineering-Engineering

\section{General Terms}

Design, Experimentation, Human Factors

\section{INTRODUCTION}

Telepresence robots are a current topic of research in $\mathrm{Hu}-$ man Robot Interaction (HRI). Telepresence itself is manifested in a large number of applications that involve interaction through video. This has originated the perception of telepresence robots as "embodied video conferencing on wheels" 5. On the other hand, telepresence has been a topic traditionally associated to virtual and mixed reality. In this context, virtual worlds offer an alternative environment for human-robot interaction in telepresence scenarios. In this paper we present PAC4, a system that connects virtual worlds with real robots allowing virtual world users to access and use the robot capabilities in a telepresence scenario. We also present a user experiment conducted to evaluate the effects of PAC4 on the feeling of social presence experienced by virtual world users.

\section{PAC4 DESIGN AND IMPLEMENTATION}

PAC4 implements the observer design pattern, a "one-tomany dependency between objects so that when one object changes state, all its dependents are notified and updated automatically" 3,2 .

A typical process flow in PAC4 starts when a new virtual or real agent makes its various services available within the

Copyright is held by the author/owner(s).

HRI'12, March 5-8, 2012, Boston, Massachusetts, USA.

ACM 978-1-4503-1063-5/12/03. system. Agents identify themselves to PAC4 by providing a Universal Resource Identifier (URI). PAC4 checks for the type of the agent and services that are being published. If it is a robot it contacts the RoboDB semantic information database [4] requesting information about the robot capabilities. Capabilities are treated as services related to the robot. Each service is a subject to which observers -other robots, or virtual world applications- can attach. PAC4 represents the robot capabilities indicating their name and type, its inputs and outputs. PAC4 uses the robot identifier and the capability inputs and outputs names and types to establish which observers to notify about the event.

\section{EVALUATION}

For the evaluation of PAC4, we focused on the study of social presence, a key element in telepresence robotics. The experiment was setup in a health and care telepresence scenario where the virtual world user communicated with a friend or relative in a remote location. The virtual world used was Second Life. The real world component was the "apartment" where the friend or relative "lived". Figure 1 shows the real living area, and its virtual counterpart in Second Life. An iCat robot [6] was also available in the living room area of the apartment. This robot can move its head, "talk" using text to speech, respond to touches in its head and "paws", and provide audio and video streams.

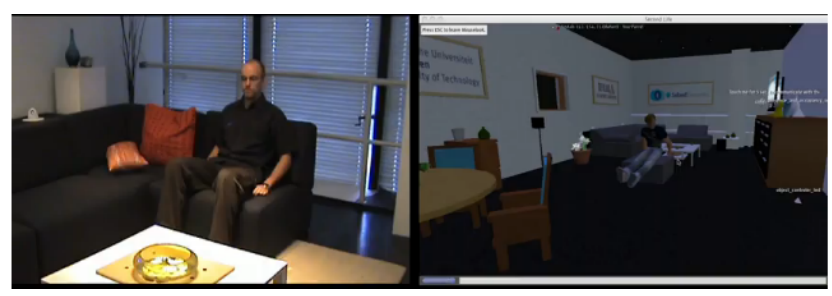

Figure 1: Real room located in the Context Lab and its virtual counterpart in Second Life.

The term PAC4 refers to a virtual world enhanced with the capabilities of real robots using the PAC4 system previously described. The term plain Second Life refers to the same virtual world without this enhancement. Additionally, the VoIP software ooVoo was used as baseline for comparison and evaluation of the overall effect of virtual worlds on the telepresence experience.

A mixed between-within subjects experiment design was used. The measurement device used was the IPO-Social 
Presence Questionnaire (IPO-SPQ) developed by De Greef \& Ijsselsteijn 11. A total of 20 participants, 8 female and 12 male with ages between 20 and 34, took part in the experiment. Ten participants were selected randomly to be placed in the PAC4 group, and ten in the plain Second Life group.

A one-way between-groups analysis of covariance was conducted to compare the effectiveness of two different communication systems designed to measure the level of social presence experienced by the participants in a remote communication scenario. The independent variable was the type of system used (PAC4, plain Second Life) and the dependent variable consisted on the scores of the IPO-SPQ questionnaire administered after the participants completed the indicated tasks with the communication systems. The estimated mean score values and standard deviation for PAC4 and plain Second Life were $M=4.000$, Std. Dev. $=0.685$ and $M=3.806$, Std. Dev. $=0.946$ respectively. The IPO-SPQ scores of the participants related to the software ooVoo were used as the covariate in this analysis. After adjusting for the covariates there were no significant differences between the two communication system groups (PAC4 and plain Second Life) on the scores for the IPO-SPQ, $F(1,17)=0.554$, $p=0.467$, eta squared $=0.032$. There was a moderate relationship between the covariate and the independent variable a indicated by an eta squared $=0.054$.

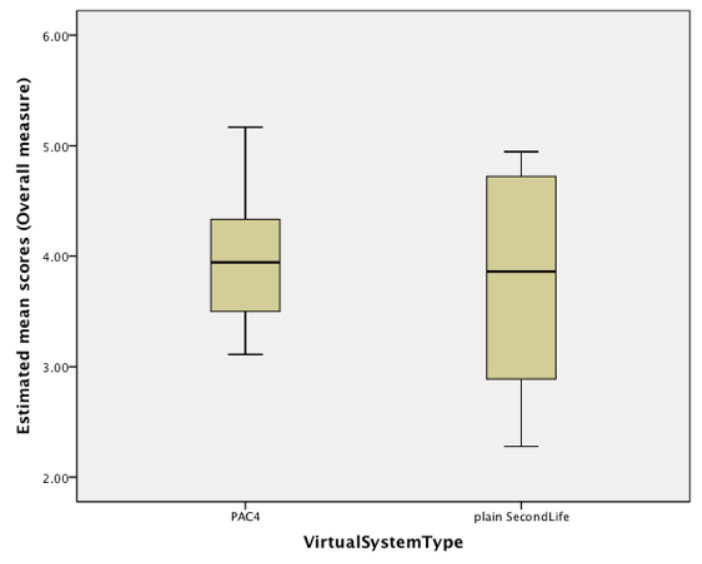

Figure 2: Mean presence scores of PAC4 vs plain Second Life

The analysis of the video recordings taken during the experiment and the responses to open-ended questions, showed divided opinion over the benefits of PAC4 over Second Life. Most participants stated that they felt "more connected" to the remote peer, and that PAC4 provides "better information about the other person" than the VoIP software. However, several mentioned that there might be issues with privacy as "I can see information about the other person even when the person is not online or chatting with me".

\section{DISCUSSION}

The results indicate that although the participants appreciate the potential of PAC4 as an enabling technology for telepresence, they perceive a large overhead and complexity in the virtual world software. Particularly, participants mentioned that alternative means of communication like VoIP software (e.g. skype, ooVoo, etc.) were simpler to understand, faster, and in general, more familiar to them. Some participants went further to comment that although they value the potential of telepresence using a robot for health and care situations, moving around in the virtual world, and talking to others through an avatar was complicated and unnatural.

All this seems to suggest that in general, virtual worlds technology is not mature enough to allow for meaningful interactions and applications where efficient telepresence is needed, making users feel that virtual worlds are more a hindrance than a facilitator. In fact, the participants indicated that although having a robot they could remotely interact with helped them to feel more connected and more aware of what happened in the remote location, they felt that they were equally present when they could talk to the remote person without the mediation of a virtual representation.

\section{CONCLUSION}

This paper introduced the PAC4 system that connects virtual worlds and real robots, enabling virtual world users to access the robot's capabilities from within the virtual world. A user experiment was conducted to evaluate the effect of PAC4 on telepresence. The results of the evaluation of PAC4 should be seen under the correct light. Although the questionnaire scores indicate that PAC4 indeed improves the level of presence with respect to plain Second Life, the fact that the overall evaluation of virtual worlds by the participants was not positive produced a negative effect in the overall appreciation of any virtual world-based software. In other words, there seems to be an indication that virtual world applications and technologies are not yet ready to compete with alternative forms of communication like the VoIP software ooVoo. This is a likely explanation as to why the results of the evaluation are not statistically significant.

\section{REFERENCES}

[1] P. De Greef and W.A. Ijsselsteijn. Social presence in a home tele-application. CyberPsychology \& Behavior, 4(2):307-315, 2001.

[2] L.M.G. Feijs. Modelling Microsoft COM using $\pi$-calculus. In Proceedings of the Wold Congress on Formal Methods in the Development of Computing Systems-Volume II, pages 1343-1363. Springer-Verlag, 1999.

[3] Erich Gamma, Richard Helm, Ralph Johnson, and John Vlissides. Design patterns: elements of reusable object-oriented software. Addison-Wesley, 1995.

[4] A. Juarez, C. Bartneck, and L. Feijs. Using semantic technologies to describe robotic embodiments. In Proceedings of the 6th international conference on Human-robot interaction, pages 425-432. ACM, 2011.

[5] K.M. Tsui, M. Desai, H.A. Yanco, and C. Uhlik. Exploring use cases for telepresence robots. In Proceedings of the 6th international conference on Human-robot interaction, pages 11-18. ACM, 2011.

[6] A. van Breemen, X. Yan, and B. Meerbeek. iCat: an animated user-interface robot with personality. In Proceedings of the fourth international joint conference on Autonomous agents and multiagent systems, pages 143-144. ACM, 2005. 